\title{
Role of esophageal manometry in polysomnography of patients with chronic respiratory failure
}

\author{
Debasis Behera ${ }^{1}$, J C Suri², Pranav Ish'², Ruchi Rekha Behera ${ }^{3}$, Shibdas Chakrabarti², \\ Viswesvaran Balasubramanian ${ }^{2}$ \\ ${ }^{1}$ Department of Pulmonary Medicine, Kalinga Institute of Medical Science, Bhubaneswar, Odisha; ${ }^{2}$ Department of \\ Pulmonary, Critical Care and Sleep Medicine, Vardhman Mahavir Medical College and Safdarjung Hospital, New Delhi; \\ ${ }^{3}$ Department of Pediatrics, Srirama Chandra Bhanja Medical College, Cuttack, India
}

\begin{abstract}
Esophageal pressure (Pes) monitoring is performed during polysomnography (PSG) with a thin, water-filled catheter connected to a transducer. The resulting quantitative assessment of respiratory effort can aid in the accurate diagnosis of sleep-related breathing disorders. This was a prospective observational study using Pes in PSG for thirty patients with chronic respiratory failure (CRF) conducted in the Department of Pulmonary, Critical Care and Sleep Medicine at a tertiary care centre of North India. Sleep scoring was done by conventional method and using esophageal manometry and compared polysomnography normal without esophageal manometry recording (PSGN) and
\end{abstract}

Correspondence: Debasis Behera, Department of Pulmonary Medicine, Kalinga Institute of Medical Science, Bhubaneswar, Odisha 751024, India.

E-mail: drdebasis8@gmail.com

Authors' contributions: All the authors made a substantive intellectual contribution. All the authors have read and approved the final version of the manuscript and agreed to be accountable for all aspects of the work.

Funding: None.

Conflict of interest: The authors declare no potential conflict of interest.

Informed consent: Written informed consent was obtained from all patients.

Availability of data and materials: All data generated or analyzed during this study are included in this published article.

Key words: Chronic respiratory failure; sleep-disordered breathing; esophageal manometry; RERA; RDI.

Received for publication: 29 December 2020.

Accepted for publication: 15 May 2021.

CCopyright: the Author(s), 2021

Licensee PAGEPress, Italy

Monaldi Archives for Chest Disease 2021; 91:1756

doi: 10.4081/monaldi.2021.1756

This article is distributed under the terms of the Creative Commons Attribution Noncommercial License (by-nc 4.0) which permits any noncommercial use, distribution, and reproduction in any medium, provided the original author(s) and source are credited. polysomnography with esophageal manometry scoring (PSGE). Apnea hypopnea index (AHI) index was similar in both groups. However, respiratory effort related arousals (RERAs) were diagnosed easily using Pes resulting in significant increase in respiratory disturbance index (RDI) and even reclassification in terms of severity of sleep apnea. Besides, Pes was also useful to distinguish obstructive from central hypopnea which cannot be distinguished by routine PSG which can help guide therapy particularly in chronic respiratory failure patients with hypoventilation. Such patients with hypoventilation often require bilevel positive airway pressure as ventilatory support. Central hypopneas and apneas with hypercapnia may require a higher-pressure support, a backup rate or even advanced volume assured modes of ventilation. Thus, it can be concluded that Pes in PSG remains a safe and generally well-tolerated procedure. Use of Pes aids to detect RERA and thereby RDI; a better marker of sleep related breathing disorder rather than AHI. It also helps in differentiating between obstructive and central hypopnea.

\section{Introduction}

Sleep-disordered breathing (SDB) describes a group of disorders of respiratory pattern or ventilation during sleep with obstructive sleep apnea (OSA) being the most common subtype. In the general population, prevalence of SDB ranges from $5 \%$ to $22 \%$. However, increased prevalence of SDB has been reported in patients with chronic respiratory failure. Some studies have reported an incidence as high as $80-90 \%$ OSA in patients with diffuse parenchymal lung disease (DPLD) and 20-40\% in chronic obstructive pulmonary disease (COPD) patients [1].

It has been recognized that electroencephalographic arousals (EEG arousals) can be caused by respiratory efforts against upper airway resistance even in the absence of an apnea or hypopnea (UARS: upper airway resistance syndrome) [2-3]. These are commonly observed in mild cases of OSA and these arousals exacerbate daytime sleepiness [4-5]. The American Academy of Sleep Medicine (AASM) Task Force has proposed that these EEG arousals be defined as respiratory effort-related arousals (RERA). In the pathophysiology of OSA, increases in negative intrathoracic pressure caused by respiratory efforts against complete or partial upper airway obstruction increases venous return to the right ventricle, afterload on the left ventricle, causing ventricular hypertrophy [6-8], and resulting in congestive heart failure. Moreover, a significant drop in blood pressure, which is called pulsus paradoxus [9], can be caused by a drop of the intrathoracic pressure which is then followed by a significant overshoot accompanied 
with arousal response. These fluctuations in blood pressure play a role in the pathogenesis of hypertension [10]. The same pathophysiology is plausible to the patients with UARS which is unfortunately an ignored entity and difficult to diagnose.

Esophageal pressure (Pes) measurement, which has generally been considered to be an accurate method for the detection and quantification of respiratory efforts and intrathoracic pressure, is not routinely carried out polysomnography (PSG). As the severity of OSAS is usually assessed in terms of the AHI instead of RDI which includes RERA, UARS is easily overlooked.

In patients with respiratory failure $\mathrm{SpO}_{2}$ lies in the steep portion of oxygen dissociation curve (ODC), where a small fall in $\mathrm{pO}_{2}$ during hypoventilation resulted in significant desaturation, falsely marking the events as hypopnea. Clear discrimination of hypopneas is also important because these events are representative of most respiratory disturbances compared with apneas [11-12]. The obstruction of the upper airway limits the flow that can be generated by respiratory muscles. Thus, ventilatory effort continuously increases to achieve sufficient flow. This increasing effort can be measured by esophageal pressure swings, which is currently the gold standard for the assessment of ventilator effort. However; the application of the esophageal pressure sensor is invasive and not widely accepted or tolerated by patients. Therefore, the measurement of the esophageal pressure (Pes) has not become routine practice, with its use often confined to experimental settings. Some investigators use diaphragmatic or intercostal electromyogram [13]. Measurement of Pes helps in differentiating obstructive hypopnea (crescendo negative increases in Pes values) from central hypopnea (no increase to decrease in Pes value).

\section{Methods}

It was a prospective observational study of SDB using esophageal manometry in patients with chronic respiratory failure (CRF) conducted in Department of Pulmonary, Critical Care, and Sleep Medicine at a tertiary care center of North India. All the patients with CRF with history suggestive of SDB were included in the study over a 12-month study period. Patients with neurological diseases, severe psychiatric diseases, taking drugs that affect sleep architecture, injury to esophagus, and age lesser than 18 were excluded from the study. Institutional Ethical Committee (IEC) clearance was taken. A valid informed consent from the patients was taken. All patients underwent a postbronchodilator spirometry [Spiroair system (Medisoft, Sorinnes, Belgium)] to confirm the diagnosis and were classified obstructive or restrictive disease. History regarding excessive daytime sleepiness, snoring, early morning headache, lethargy, and fatigue was taken and Epworth sleepiness score (ESS) was calculated. Patients with scores $\geq 11$ with sleepiness during work or driving were suspected to be having OSA. Arterial blood gas (ABG) analysis was done to confirm hypercapnic respiratory failure patients. The following information was collected from all study subjects: age, sex, occupation, past history of diabetes, hypertension, hyperlipidemia, hypothyroidism, exposure to biomass, and smoking. The body mass index (BMI) (weight in kilograms by the square of height in meters) was calculated for all subjects. Polysomnography was performed in the night using Alice 6 LDX (Philips Respironics, Murrysville, USA). In PSG, body functions including brain (EEG), eye movements (EOG), muscle activity or skeletal muscle activation (EMG), and heart rhythm (ECG) during sleep were recorded. Nasal flow during breathing, chest, and abdominal movements was recorded to iden- tify central apnea and OSA. Esophageal pressure and $\mathrm{ETCO}_{2}$ were also continuously measured to document hypoventilation. The PSG findings were scored in 30 second windows following the recommended criteria of the American Academy of Sleep Medicine (AASM) 2007 guidelines. The sleep efficiency, sleep onset time, REM latency, wake after sleep onset (WASO), percentage of time in different sleep stages, apnea index (AI), hypopnea index (HI), apnea-hypopnea index (AHI), respiratory event-related arousal (RERA), and respiratory disturbance index (RDI) were calculated.

\section{Esophageal manometry}

Assessment of intrathoracic pressure relies on the changing volume of the thoracic cavity that varies with the respiratory cycle, and it provides an indirect measurement of pleural surface pressure. During inspiration, contraction of the diaphragm and accessory muscles of respiration results in expansion of the thoracic cavity with a corresponding increase in negative pressure which, in turn, inflates the lungs. During expiration, there is a decrease in lung volume and diaphragmatic relaxation, which results in a relative positive pressure within the thoracic cavity. A fluid-filled catheter (infant feeding tube of size 6 French) was placed within the esophagus to detect transmitted variation in intrathoracic pressure, since the esophageal wall expands and contracts during the respiratory cycle, resulting in corresponding fluctuations in esophageal pressure. Increased respiratory effort associated with the increased upper airway resistance of sleep-related breathing disorders was measured by this technique. This fluid-filled catheter system for assessing respiratory effort was based on a high-pressure low-flow valve connected to the esophageal catheter and pressure transducer. A pressure infuser maintained a pressure of $300 \mathrm{mmHg}$ on a saline bag, resulting in a constant drip rate of $3 \mathrm{cc}$ per hour. The fluid in the catheter transmitted the pressure to the transducer and the continuous drip kept the catheter patent, allowing the measurement of relative changes in esophageal pressure during sleep.

\section{Scoring}

PSG done by using esophageal manometry was scored by a sleep physician without using esophageal pressure values and by another doctor using esophageal pressure values for accurate detection of RERA and to distinguish between obstructive and central hypopnea. AASM scoring manual definitions were used for scoring apnea and hypopnea. RERA calculated by esophageal manometry were added to AHI to derive the RDI. The AHI and RDI was used to grade the degree of severity of SDB (5-14 was regarded as mild, 15-30 regarded as moderate, and greater than 30 as severe). RDI using esophageal manometry was compared with that of conventional scoring. RERA was scored if there is a sequence of breaths lasting at least 10 seconds characterized by increasing respiratory effort or flattening of the nasal pressure waveform leading to an arousal from sleep when the sequence of breaths does not meet criteria for an apnea or a hypopnea. In RERA, Pes crescendos may occur independent of obstructive hypopneas or apneas and may lead to an EEG arousal from sleep. Alveolar hypoventilation was defined as an increase in the $\mathrm{PaCO}_{2}$ to $>55 \mathrm{mmHg}$ for $\geq 10$ minutes, or an increase in the $\mathrm{PaCO}_{2}$ by $\geq 10 \mathrm{mmHg}$ above the awake supine value to a value over $50 \mathrm{mmHg}$ for $\geq 10$ minutes.

\section{Statistical analysis}

Categorical variables were presented in number and percentage $(\%)$ and continuous variables were presented as mean \pm SD and median. Statistical tests were applied as follows-quantitative variables were compared using paired $\mathrm{T}$ test between polysomnography 
normal without esophageal manometry recording (PSGN) and polysomnography with esophageal manometry scoring (PSGE), Qualitative variables were correlated using Chi-Square test. A p value of $<0.05$ was considered statistically significant. The data was entered in MS EXCEL spreadsheet and analysis was done using Statistical Package for Social Sciences (SPSS) version 21.0.

\section{Results}

Thirty chronic respiratory failure patients with daytime hypercapnia and a history suggestive of sleep disordered breathing were included in the study. It was a predominantly young adult male (70\%) population with mean age of 45 years with history of smoking in $60 \%$ of the population and a mean ESS of 14.5. On comparing PSG scoring, it was found that that PSGE helped to identify RERA which altered the RDI significantly. The RDI increased from 13.4 in PSGN group to 21.45 in PSGE group (Table 1). Besides being statistically significant, this altered the severity classification of the illness. Moderate severity group increased from $23.33 \%$ to $43.33 \%$ and severe group had a rise from $20 \%$ to $33.33 \%$ (Figure 1). There was no difference in number of apneas (both obstructive and central) between PSGN and PSGE group, as apneas can be easily distinguished (whether obstructive or central) by normal polysomnography scoring method as described in AASM manual. So, AHI index remained unchanged in both the methods, but RERAs can be diagnosed easily using esophageal manometry resulting in increase in RDI (Table 2). Esophageal manometry pressure also helped to distinguish between obstructive and central hypopnea and 7 out of 30 hypopnea events were reclassified as central hypopnea using PSGE. This differentiation does not change the RDI overall but can have therapeutic implication especially in chronic hypercapnic respiratory failure patients who often have hypoventilation in sleep. The mean rise in $\mathrm{ETCO}_{2}$ was $13.6 \mathrm{mmHg}$ and hypoventilation was documented in all the thirty patients included in the study.

\section{Discussion}

To the best of our knowledge, this is one of the first studies using Pes in India to evaluate PSG. In our study we found among 30 patients of chronic respiratory failure 27 had SDRB (3-normal, 7-mild, 13-moderate, 10-severe) when scoring was done without using Pes values. When scoring was done using Pes values then we found there is both increased number and severity of disease among those 30 patients (0- normal, 7-mild, 13-moderate, 10severe). This increased number and severity of disease was due to increased detection of RERAs using Pes. The American Academy of Sleep Medicine (AASM) Task Force has proposed fluctuations

\section{COMPARISION OF RDI SEVERITY B/W PSGN \& PSGE}

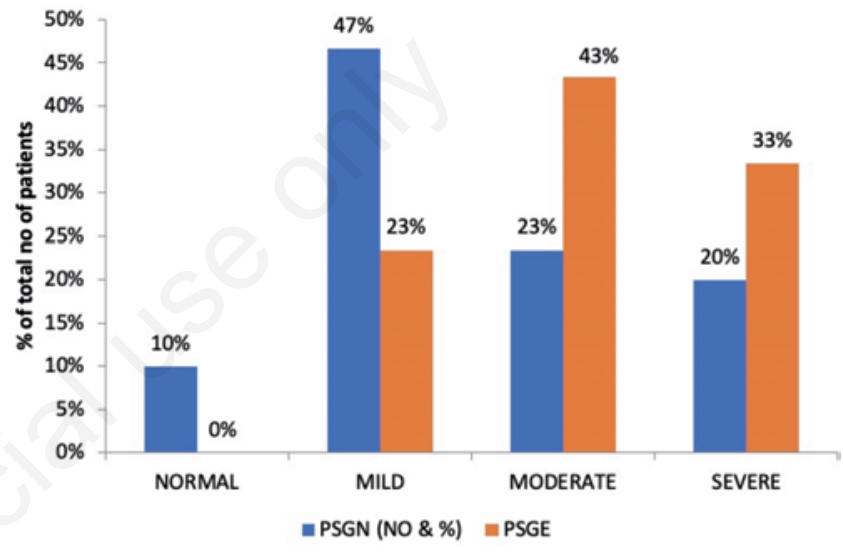

Figure 1. RDI in PSGN \& PSGE groups. PSGN, polysomnography normal (events scoring done without using esophageal manometry); PSGE, polysomnography esophageal manometry (events scoring done using esophageal manometry).

Table 1. Comparison of respiratory events in polysomnography normal without esophageal manometry recording (PSGN) and polysomnography with esophageal manomentry scoring (PSGE) groups.

\begin{tabular}{lccc} 
Events (Median and IQR) & PSGN & PSGE value \\
RERA NO & $7.5(0-63)$ & $38.5(12-179)$ & $<0.0001$ \\
RERA INDEX & $1.55(0-13)$ & $7.35(2.8-29.5)$ & $<0.0001$ \\
\hline RD NO & $67.5(18-387)$ & $105.5(31-410)$ & $<0.0001$ \\
RD INDEX & $13.4(4-73.6)$ & $21.45(6.6-78)$ & $<0.0001$ \\
\hline SEVERITY RDI & $1(0-3)$ & $2(1-3)$ & 0.0001 \\
\hline
\end{tabular}

PSGN: polysomnography normal without esophageal manometry recording; PSGE: polysomnography with esophageal manometry scoring; HV: hypoventilation; H: hypopnea; RERA: respiratory effort related arousal; RD: respiratory disturbances; RDI: respiratory disturbance index.

Table 2. Comparison of respiratory disturbance index (RDI) severity between polysomnography normal without esophageal manometry recording (PSGN) and polysomnography with esophageal manomentry scoring (PSGE).

\begin{tabular}{lccc} 
Severity RDI & PSGN $($ NO and $\%)$ & PSGE $($ NO and $\%)$ & \\
NORMAL & $3(10.00 \%)$ & $0(0.00 \%)$ & 0.043 \\
MILD & $14(46.67 \%)$ & $7(23.33 \%)$ & $13(43.33 \%)$ \\
MODERATE & $7(23.33 \%)$ & $10(33.33 \%)$ & \\
SEVERE & $6(20 \%)$ & & \\
\hline
\end{tabular}

PSGN: polysomnography normal without esophageal manometry recording; PSGE: polysomnography with esophageal manometry scoring; RDI: respiratory disturbance index. 
in blood pressure caused by RERA can play a role in the pathogenesis of hypertension and other comorbidities ${ }^{10}$ in OSAS. Detecting and treating such events in these patients may help to decrease morbidity and mortality. Besides patients being reclassified from mild to moderate severity of sleep apnea is clinically relevant as all moderate and severe patients of sleep apnea need PAP therapy. Thus, RDI can serve as a better tool to determine severity and initiate therapy for sleep apnea. AASM guidelines recommend calculation of AHI, but state that calculating RDI as optional as unfortunately detecting RERA is challenging with a routine PSG. In the absence of Pes, a flattening of nasal flow or increased respiratory effort for 10 seconds not meeting the criteria for apnea or a hypopnea is marked as RERA. This is very subjective and difficult. However, with the use of Pes, increased respiratory effort can easily be detected by a negative fall in esophageal pressure. The clinical significance of RERA has long been debated, but studies have shown an increased risk of hypertension and cardiovascular comorbidities [14].

Besides, Pes helps to identify central hypopnea and differentiate from obstructive hypopnea. In the absence of Pes, snoring, thoracoabdominal paradox and flattening of flow are subtle signs for obstructive hypopnea. However, the assessment of increase in effort by Pes on obstructive apnea makes scoring objective. AASM guidelines state this differentiation to be optional as usually hypopnea is corrected by CPAP itself. However, in patients of hypoventilation, central hypopneas with hypercapnia require a higher inspiratory PAP, back up rate ( $\mathrm{S} / \mathrm{T}$ modes) or sometimes even advanced modes of volume assured ventilation [15]. Thus, it is prudent to diagnose and differentiate such events for optimal titration.

\section{Conclusions}

Our study contributes to the limited literature on the utility of esophageal pressure monitoring during PSGs and is relevant because PSGs were conducted with use of the current technical specifications outlined by AASM. Esophageal pressure monitoring remains a safe and generally well-tolerated procedure, although expertise in patient selection, placement, and interpretation are needed. Use of esophageal manometry is definitely indicated in patients giving history suggestive of SDRB in spite of having normal AHI for detection of RERAs, hence RDI is a better indicator of SDRB rather than AHI.

\section{References}

1. Romem A, Iacono A, McIlmoyle E, et al. Obstructive sleep apnea in patients with end stage lung disease. J Clin Sleep Med 2013;9:687-93.

2. Exar EN, Collop NA. The upper airway resistance syndrome. Chest 1999;115:1127-39.

3. Guilleminault C, Stoohs R, Clerk A, et al. A cause of excessive daytime sleepiness: the upper airway resistance syndrome. Chest 1993;104 781-7.

4. Mikami A, Watanabe T, Motonishi M, et al. Frequent breathing-related electroencephalogram arousals in four patients with mild obstructive sleep apneas. Psychiatry Clin Neurosci 1999;53:307-9.

5. Fietze I, Quispe-Bravo S, Schiller W, et al. Respiratory arousals in mild obstructive sleep apnea syndrome. Sleep 1999;22:583-9.

6. Redline S, Strohl KP. Recognition and consequences of obstructive sleep apnea hypopnea syndrome. Clin ChestMed 1998;19:1-19.

7. Berman EJ, DiBenedetto RJ, Causey DE, et al. Right ventricular hypertrophy in patients with newly diagnosed obstructive sleep apnea. Chest 1991;100:347-50.

8. Salejee I, Tarasiuk A, Reder I, Scharf SM. Chronic upper airway obstruction produces right but not left ventricular hypertrophy in rats. Am Rev Respir Dis 1993;148:1346-50.

9. Shiomi T, Guilleminault C, Stoohs R, Schnittger I. Leftward shift of the interventricular septum and pulsus paradoxus in obstructive sleep apnea syndrome. Chest 1991;100:894-902.

10. Morgan RJ, Dempsey JA, Pegelow DF, et al. Blood pressure perturbations caused by subclinical sleep-disordered breathing. Sleep 1998;21:737-46.

11. Galetke W, Randerath WJ, Feier C, et al. Esophageal pressure method and impulse oscillometry to assess mechanical properties of the respiratory system in healthy men. Med Sci Monit 2009; 15:CR429-35.

12. Sleep-related breathing disorders in adults: recommendations for syndrome definition and measurement techniques in clinical research. The report of an American Academy of Sleep Medicine Task Force. Sleep 1999;22:667-89.

13. Luo YM, Tang J, Jolley C, et al. Distinguishing obstructive from central sleep apnea events: diaphragm electromyogram and esophageal pressure compared. Chest 2009;135:1133-41.

14. Ogna A, Tobback N, Andries D, et al. Prevalence and clinical significance of respiratory effort-related arousals in the general population. J Clin Sleep Med 2018;14:1339-45.

15. Chakrabarti S, Ish P, Rathi V. OSA and COPD overlap syndrome - double trouble. J Adv Res Med 2018;5:1-6. 